\title{
Highly Sensitive Sizing Response Induced by 2-Bromination of Fatty Acids and their Pairing with Anchor Sites Formed on Paper Surfaces
}

\author{
Takuya Kitaoka, Nobuo Tanaka, Kana Matsuyama, and Hiroki Yamamoto
}

\author{
Department of Agro-environmental Sciences, Graduate School of Bioresource and Bioenvironmental \\ Sciences, Kyushu University, 6-10-1 Hakozaki, Higashi-ku, Fukuoka 812-8581, Japan
}

\begin{abstract}
The base handsheets were prepared from a pulp suspension containing either aluminum sulfate (alum) or polydiallyldimethylammonium chloride (PDADMAC), followed by being soaked in the chloroform solution of stearic and palmitic acids (SPA), which were brominated, or non-brominated, at the $\alpha$-carbon positions adjacent to respective carbonyl groups. No sizing effect was observed in the absence of alum or PDADMAC, and thus these additives that form anchor sites for size components were indispensable for sizing appearance. Besides, extremely different sizing responses were found, strongly depending on the combinations of fatty acids and additives. Alum-treated handsheets showed good sizing effects by dipping them in an SPA/chloroform solution; however sizing with 2-brominated SPA (Br-SPA) had no contribution to sizing appearance. By contrast, PDADMAC-handsheets sized with Br-SPA exhibited high sizing degrees, although there was no sizing effect in the case of using non-brominated, original SPA. Highly sensitive sizing responses would provide a new insight to elucidate paper sizing mechanisms.
\end{abstract}

(Received 24 May, 2010 ; Accepted 11 July, 2010)

\section{Introduction}

Paper sizing enables a controllable delay of liquid penetration into a porous network structure composed of hydrophilic pulp fibers [1]. Especially, water repellency is one of the most significant properties in the practical applications of paper products, and sizing mechanisms have been a main issue in paper chemistry and industry. In particular, rosin acid/aluminum sulfate (alum) systems have long and widely been applied, and a variety of researches on rosin-alum sizing mechanisms have been reported with regard to chemical reactions of rosin with alum [2-6], retention behavior [7-10], and distribution on paper sheets [11-16].

Rosin size is a mixture of amphipathic diterpenes of hydrophobic aromatic ring and hydrophilic carboxyl group, and demonstrate high sizing performance only in the combination with alum. Thus, a specific interaction at the carboxyl group/aluminum component interface has become the center of attraction for rosin-related sizing mechanism. Fatty acid mixture, stearic and palmitic acids (SPA) with an equal weight, is useful model components having hydrophobic alkyl chain and one carboxyl terminus for the fundamental investigation of rosin sizing systems $[5,6,15,16]$. In our previous study, a direct detection of specific attraction between carboxyl group and oxidized aluminum was achieved by atomic force microscopy, and such a unique interaction was successfully applied to the waterproofing of hydrophilic porous glass filter [17].

The present study reports a new discovery of very sensitive sizing responses that strongly depend on the combinations of size components (SPA or 2-brominated SPA ; Br-SPA) and anchor sites (alum and polydiallyldimethylammonium chloride: PDADMAC) preformed on paper surfaces. Bromination of $\alpha$-carbon adjacent to carbonyl group eventually induced extremely different sizing effects in the SPA-mediated sizing systems.

\section{Experimental}

\subsection{Materials}

A commercial hardwood bleached kraft pulp was beaten to $450 \mathrm{~mL}$ of Canadian Standard Freeness [18] with a Hollander beater [19], for handsheet-making. A fatty acid mixture consisting of stearic and palmitic acids (Wako Pure Chemical Industries, Co. Ltd.) in an equal weight was used as a model component of rosin size [15]. 2-Brominated stearic and palmitic acids were obtained from the supplier, and used without further purification. Aluminum sulfate $\left(\mathrm{Al}_{2}\left(\mathrm{SO}_{4}\right)_{3}\right.$, alum), PDADMAC (molecular weight: $c$ a. $3 \times 10^{5}$; charge density: $5.5 \mathrm{meq} / \mathrm{g}$ ), and other chemicals were of pure reagent grade (Sigma-Aldrich, Co. Ltd.), and used as purchased. 
The water used in this study was purified with a Milli-Q system (Millipore, Inc.).

\subsection{Sizing treatment}

Either alum $(0-2.0 \%$ on dry weight of pulp) or PDADMAC ( $0-0.2 \%$ on dry pulp) was added to a $0.15 \%$ pulp suspension ( $\mathrm{pH}$ 6.5), and the handsheets with a basis weight of $60 \mathrm{~g} / \mathrm{m}^{2}$ were prepared according to TAPPI standard methods [20]. The conditioned handsheets were soaked in either a $0.25 \%(\mathrm{w} / \mathrm{v}) \mathrm{SPA} /$ chloroform $\left(\mathrm{CHCl}_{3}\right)$ solution or a $0.50 \%(\mathrm{w} / \mathrm{v}) \mathrm{Br}-\mathrm{SPA} / \mathrm{CHCl}_{3}$ solution for 1 min, and then air-dried at $23^{\circ} \mathrm{C}$ and $50 \%$ relative humidity $(\mathrm{RH})$ for at least $24 \mathrm{~h}$. Glass filter (GA55; Advantec MFS, Inc.) was dipped in $0.5 \%$ alum solution and then sodium hydroxide solution was added dropwise to precipitate oxidized aluminum compounds on the fiber surfaces [17]. On the other hand, the glass filter was soaked in $0.1 \%$ PDADMAC solution, and air-dried. Each treated filter was subjected to the sizing treatment with either $0.50 \%(\mathrm{w} / \mathrm{v}) \mathrm{SPA} / \mathrm{CHCl}_{3}$ or $1.0 \%(\mathrm{w} / \mathrm{v}) \mathrm{Br}-\mathrm{SPA} /$ $\mathrm{CHCl}_{3}$ solution.

\subsection{Characterization}

Elemental analysis of the surfaces of handsheets and glass filters was carried out by X-ray photoelectron spectroscopy (XPS, AXIS-HSi; Shimadzu/Kratos, Co. Ltd.) equipped with a monochromatic AlK $\alpha$ X-ray source (1486.6 eV). All XPS measurements were performed using $15 \mathrm{kV}$ voltage and $10 \mathrm{~mA}$ current, and the pressure in the analyzing chamber was maintained below $0.5 \mu \mathrm{Pa}$. The pass energy and step width for the survey scan were set at $80 \mathrm{eV}$ and $1 \mathrm{eV}$, respectively. For narrow scans, those parameters were set at $10 \mathrm{eV}$ and $0.05 \mathrm{eV}$. The binding energies for all spectra were referenced to a $\mathrm{C} 1 \mathrm{~s}$ signal (reduced C-C) at $285.0 \mathrm{eV}$. The atomic ratio of carbon from size to cellulose on a pulp matrix, indicating the surface abundance of SPA components present on the pulp fibers, was defined by dividing the amounts of unoxidized $(\mathrm{C} 1,285.0 \mathrm{eV})$ and carbonyl $(\mathrm{C} 4,289.3 \mathrm{eV})$ carbons by those of $\mathrm{C}-\mathrm{O}(\mathrm{C} 2,286.7 \mathrm{eV})$ and O-C-O (C3, $288.1 \mathrm{eV}$ ) originating from cellulosic carbons $[15,16]$. The $\mathrm{C} 1 \mathrm{~s} / \mathrm{Si} 2 \mathrm{p}$ ratios indicating the surface size abundance on a glass filter were determined in a similar manner [17]. The sizing degrees of handsheets were evaluated by a conventional Stöckigt sizing test [21]. The contact angle (CA) of water droplets on the glass filter surfaces was measured by the sessile drop technique using a DropMaster 500 (Kyowa Interface Science, Co. Ltd.) [22]. The CA measurements were repeated at least five times for each sample $\left(25^{\circ} \mathrm{C}, 50 \% \mathrm{RH}\right)$.

\section{Results and discussion}

\subsection{Sizing behavior of paper sheets treated with fatty acids/chloroform systems}

In our previous studies $[15,17]$, we have reported that alum-pretreatment of base handsheets is indispensable for good sizing appearance, regardless of size contents, even in nonaqueous $\mathrm{SPA} / \mathrm{CHCl}_{3}$ system. Fig. 1 follows such results; both sizing effect and surface size abundance increased with an increase in the internal addition of alum in the handsheet-making process, while no sizing effect appeared in the alum-free handsheet. However, in the case of $\mathrm{Br}-\mathrm{SPA} / \mathrm{CHCl}_{3}$, nearly no sizing effect was observed in the dose range of $0-2.0 \%$ alum addition, and then the size components were poorly retained on the fiber surfaces as estimated by XPS analysis. Thus, the strong attraction between SPA and aluminum components must exist and promote the sizing effect; on the other hand Br-SPA had no interaction with aluminum sites on the fibers, resulting in the poor sizing effect.

However, such a clear relationship was completely reversed in the case of using PDADMAC as an anchor site formed on pulp surfaces. Fig. 2 compares the sizing degrees and surface size abundance of PDADMACadded paper sheets after sizing treatments with either SPA or Br-SPA. Evidently, SPA had no interaction with PDADMAC from the XPS results; however Br-SPA demonstrated strong attraction with PDADMAC on the

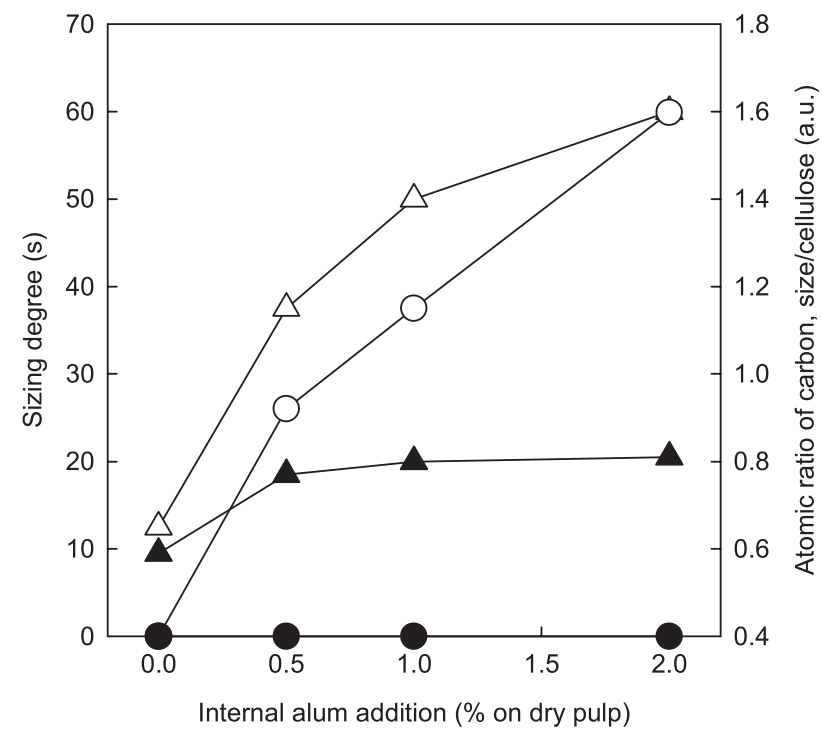

Fig. 1 Sizing degree (circles) and atomic ratio of carbon (triangles) of handsheets sized with $0.25 \%$ (w/v) $\mathrm{SPA} / \mathrm{CHCl}_{3}$ solution (open) or with $0.50 \%(\mathrm{w} / \mathrm{v}) \mathrm{Br}-\mathrm{SPA} / \mathrm{CHCl}_{3}$ solution (closed). The handsheets were prepared with $0-2.0 \%$ internal alum addition. 
fiber surface, resulting in higher size abundance and sizing effect. Cationic polyelectrolyte, PDADMAC, in general acts only as a retention aid in rosin sizing system ; but makes no contribution to sizing appearance. Thus, the experimental result for the combination of SPA and PDADMAC is reasonable. 2-Bromination of fatty acids presumably enhances the electrophilicity of $\alpha$-carbon, but seems not to be involved in the reactivity of PDADMAC molecules. At any rate, essential relations of SPA/alum and Br-SPA/PDADMAC were confirmed for good sizing.

\subsection{Water repellency of porous inorganic fiber material (glass filter)}

Strict pairing between fatty acids and anchor sites for sizing was verified by imparting similar water repellency to other fiber-network materials, e.g. a glass filter. As referred to our previous study [17], glass filters were treated with alum or PDADMAC, followed by being soaked in nonaqueous fatty acids $/ \mathrm{CHCl}_{3}$; surface size abundance and water-repellent property were determined by XPS and CA measurements. Fig. 3 shows the CA values of water droplets and $\mathrm{C} 1 \mathrm{~s} / \mathrm{Si} 2 \mathrm{p}$ atomic ratios on the sized glass mats. Very clear, highly sensitive sizing responses were also observed in the same manner as those of sized paper sheets. The CA value of SPA-sized alumtreated glass filter reached up to $140^{\circ}$, while high water absorptivity (CA $\ 0^{\circ}$ ) was observed in the case of BrSPA although $\mathrm{C} 1 \mathrm{~s} / \mathrm{Si} 2 \mathrm{p}$ ratios were almost identical. When the $\mathrm{Br}-\mathrm{SPA} / \mathrm{CHCl}_{3}$ solution was subjected to PDADMAC-treated glass filters, distinct hydrophobicity

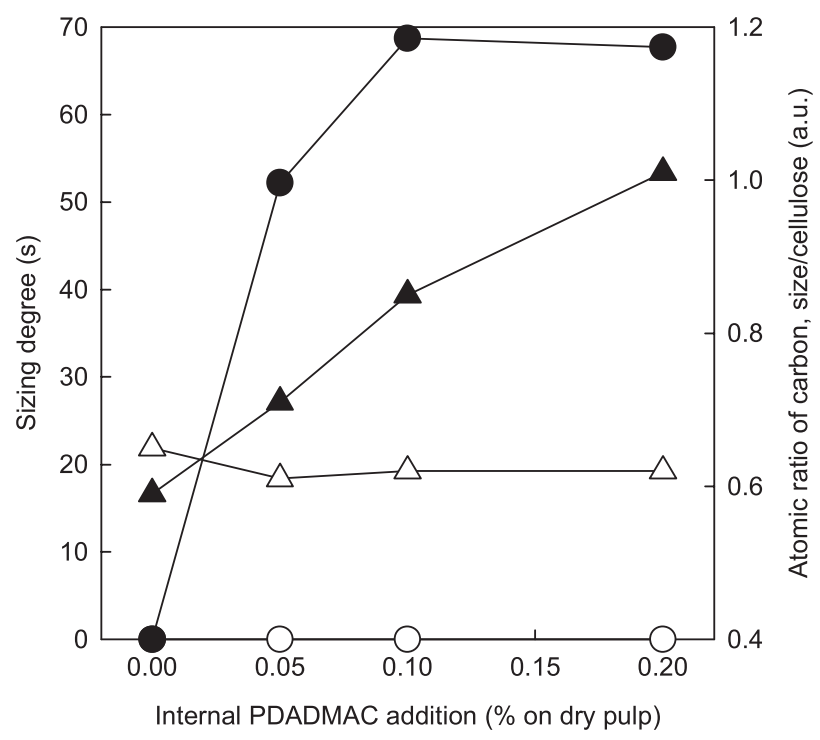

Fig. 2 Sizing degree (circles) and atomic ratio of carbon (triangles) of handsheets sized with $0.25 \%$ (w/v) $\mathrm{SPA} / \mathrm{CHCl}_{3}$ solution (open) or with $0.50 \%(\mathrm{w} / \mathrm{v}) \mathrm{Br}-\mathrm{SPA} / \mathrm{CHCl}_{3}$ solution (closed). The handsheets were prepared with $0-0.2 \%$ internal PDADMAC addition. was determined $\left(\mathrm{CA}>130^{\circ}\right)$; however $\mathrm{C} 1 \mathrm{~s} / \mathrm{Si} 2 \mathrm{p}$ ratio was on a lower level as compared to Br-SPA/alum system. On the other hand, large amounts of SPA components on the PDADMAC-treated filters had no contribution to water repellency $\left(\mathrm{CA} \bigotimes 0^{\circ}\right)$. These results strongly suggested the specific pairing rules for the slowdown mechanism of water penetration for porous fiber-network structures, regardless of size retention. An attractive force at the carboxyl terminus of SPA/oxidized aluminum interface as reported [17] must be an essential factor for the SPA-sizing appearance, but 2-bromination markedly eliminated such significant effect. By contrast, Br adduct made a clear contribution to sizing appearance of PDADMAC-treated glass fiber mats.

\subsection{Possible interactions to stimulate sizing effect}

The attractive force at the interface of carboxyl group of SPA and aluminum oxide was directly determined by force-curve measurement using atomic force microscopy [17], and is presumably allowed to stably settle the SPA components on alum-added paper surfaces. If 2-bromo $\omega$-thiol fatty acids are commercially available or can be synthesized, the direct force-curve analysis would be a powerful tool for the elucidation of sizing mechanism, and probably little interactive force may be detected. However, PDADMAC-treated surface is not suitable for the force-curve measurement due to its very sticky property under aqueous analytical conditions.

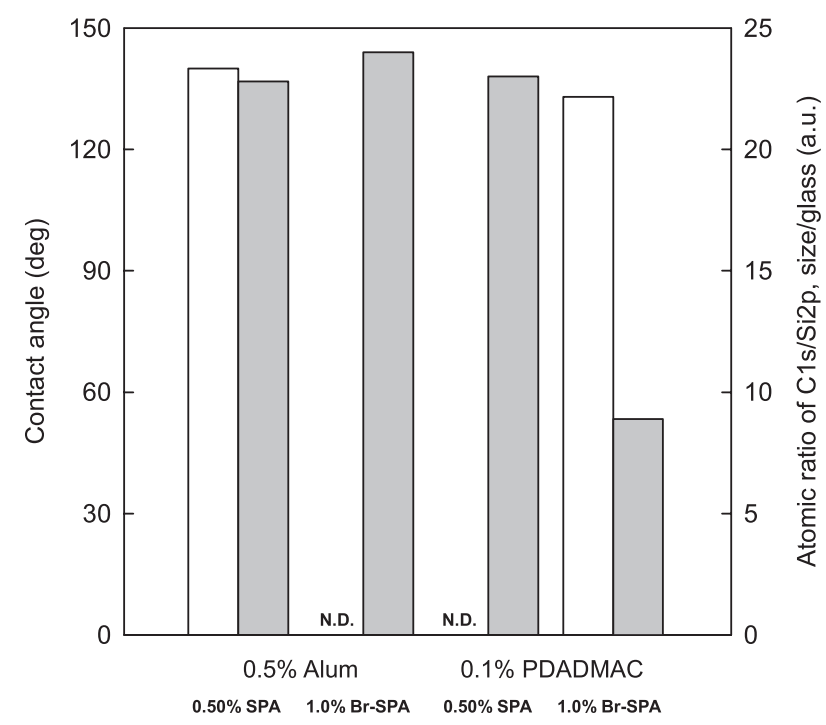

Fig. 3 Water CA value (open bars) and atomic ratio of $\mathrm{C} 1 \mathrm{~s} / \mathrm{Si} 2 \mathrm{p}$ (gray bars) on the surfaces of glass filters sized with $0.50 \%(\mathrm{w} / \mathrm{v}) \quad \mathrm{SPA} / \mathrm{CHCl}_{3}$ solution or with $1.0 \%(\mathrm{w} / \mathrm{v}) \mathrm{Br}-\mathrm{SPA} / \mathrm{CHCl}_{3}$ solution. The glass filter mats were pre-soaked in $0.5 \%$ alum solution, followed by adding sodium hydroxide solution [17] or in $0.1 \%$ PDADMAC solution, followed by air-drying. 
Theoretical consideration provides several possible mechanisms for pairings of sizes and anchor components. Negatively charged aluminum oxide interface in oil was lubricated by fatty acid moieties whose undissociated polar groups were attached to the aluminum oxide layers [23]. Formation of self-assembled monolayers of fatty acids on aluminum oxide surfaces was reported [24]; these may be involved in a specific interfacial attraction and sizing effect in the SPA/alum system. Aluminum oxides formed on pulp fibers also have a negative charge [25]. By contrast, there was no specific interaction between free carboxyl group of SPA and quaternary (positive) amine of PDADMAC; but the 2-bromination of fatty acids evidently promotes the virtual dissociation of carboxyl groups due to the electron withdrawing ability of $\mathrm{Br}$, resulting in lower $\mathrm{pKa}$ [26], which eventually enhances their negative charge character even in the nonaqueous system. Thus, some interfacial stabilization of Br-SPA with PDADMAC possibly made a contribution to clear sizing appearance, while an undesirable repulsion behavior occurred on the negatively-charged aluminum oxide surfaces. Detailed mechanisms remain unclear; but such obvious, highly sensitive sizing response would open up a new avenue for fundamental sizing chemistry and practical paper industry.

\section{Conclusion}

Strict combinations of normal or 2-brominated fatty acids with anchor components, alum or PDADMAC, respectively, on fiber surfaces were found out for good sizing (water-repellent) properties of organic paper and inorganic filter. It was suggested that the 2-bromination of fatty acids had decisive impacts on sizing appearance, in concert with a drastic change in electron circumstance around carboxyl groups. Such unique sizing phenomena will provide new conception for advanced sizing systems.

\section{Acknowledgement}

This research was supported by a Grant-in-Aid for Young Scientists (S: 21678002) from the Ministry of Education, Culture, Sports, Science and Technology, Japan (T.K).

\section{References}

1. J. M. Gess, "Paper Chemistry, second edition”, (J. C. Roberts Ed.), Chapman \& Hall, New York, p. 120 (1996).

2. E. J. Vandenberg and H. M. Spurlin, Tappi J., 50, 209 (1967).

3. R. W. Davison, J. Pulp Pap. Sci., 14, J151 (1988).

4. T. Kitaoka, A. Isogai, and F. Onabe, Nord. Pulp Pap. Res.J., 12, 26 (1997).

5. T. Kitaoka, A. Isogai, F. Onabe, and N. Suguri, Nord. Pulp Pap. Res. J., 12, 182 (1997).

6. T. Kitaoka, A. Isogai, F. Onabe, and T. Endo, Nord. Pulp Pap. Res. J., 16, 96 (2001).

7. Y. Ishida, H. Ohtani, T. Kano, S. Tsuge, and T. Yano, Tappi J., 77, 177 (1994).

8. T. Kitaoka, A. Isogai, and F. Onabe, Nord. Pulp Pap. Res.J., 10, 253 (1995).

9. K. Ito, A. Isogai, and F. Onabe, J. Pulp Pap. Sci., 25, 222 (1999)

10. F. Wang and H. Tanaka, J. Appl. Polym. Sci., 78, 1805 (2000).

11. T. Kitaoka, A. Isogai, and F. Onabe, Nord. Pulp Pap. Res. J., 15, 177 (2000).

12. F. Wang, H. Tanaka, T. Kitaoka, and M. A. Hubbe, Nord. Pulp Pap. Res. J., 15, 416 (2000).

13. M. Kato, A. Isogai, and F. Onabe, J. Wood Sci., 46, 75 (2000)

14. T. Kitaoka, H. Yamamoto, R. Nishino, and H. Tanaka, Sen'i Gakkaishi, 57, 212 (2001).

15. H. Yamamoto, T. Kitaoka, and H. Tanaka, Sen'i Gakkaishi, 59, 266 (2003).

16. H. Yamamoto, T. Kitaoka, and H. Tanaka, J. Pulp Pap. Sci., 30, 136 (2004).

17. S. Yokota, K. Matsuyama, H. Yamamoto, T. Kitaoka, and H. Wariishi, Sen'i Gakkaishi, 65, 332 (2009).

18. TAPPI Test Methods, T227 om-94 (1995).

19. TAPPI Test Methods, T200 om-89 (1995).

20. TAPPI Test Methods, T205 om-88 (1995).

21. JIS Methods, P 8122 (2004).

22. K. Matsuyama, S. Yokota, T. Kitaoka, and H. Wariishi, Sen'i Gakkaishi, 62, 89 (2006).

23. R. S. Timsit and C. V. Pelow, J. Tribol., 114, 150 (1992).

24. Y.-T. Tao, J. Am. Chem. Soc., 115, 4350 (1993).

25. T. Kitaoka and H. Tanaka, J. Wood Sci., 48, 38 (2002).

26. J. E. Katon and D. Sinha, Appl. Spectrosc., 25, 497 (1971). 